Leff, J. (200I) Why is care in the community perceived as a failure? British Journal of Psychiatry, 179, 38I-383.

E. Hoencamp Parnassia Psycho-Medisch Centrum, Monsterseweg 83, 2553 RJ, The Hague, The Netherlands

Author's reply: Regrettably, Dr Hoencamp has misinterpreted a number of phrases in my editorial. Rather than calling for increasingly restrictive legislation, I was warning the reader against this alarming consequence of public and governmental misperceptions of care in the community. On another point, I certainly did not mean to imply that closing psychiatric hospitals is itself an indication of a successful policy. The evidence of success to which I was referring consists of the growing body of research showing that the quality of life of discharged long-stay patients is improved by relocation in community homes (e.g. Leff \& Trieman, 2000). Dr Hoencamp is of course right that in-patient facilities will continue to be needed, but there is no reason for them to be located in the outdated structures of the psychiatric hospitals. There are undoubtedly problems with admission wards in district general hospitals, but these can be remedied by improved architectural design and the provision of alternatives such as acute day hospitals (Creed et al, 1990).

Although many asylums were deliberately built outside of towns, urban expansion brought them within the ambit of residential areas. Even those that remained remote, engendered in the public mind the image of life-long incarceration. I agree with Dr Hoencamp that more should be done to publicise community mental health services. We should be proud of what has been achieved and promote a high visibility. He raises the issue of the diversity of psychiatric disorders and the difficulty the public and the media have in distinguishing them. This dilemma faces any organisation attempting to change public attitudes towards people with mental illness and the services they need. The Royal College of Psychiatrists' campaign 'Changing Minds: Every Family in the Land' addresses a wide range of psychiatric disorders, while the World Psychiatric Association's 'Global Campaign against the Stigma of Schizophrenia' focuses on that one condition. Hopefully the results of these programmes will indicate which is the more effective strategy. However, early results from the
World Psychiatric Association campaign indicate that education aimed at teenagers in schools produces the most positive change in attitudes. A good strategy would seem to be the inclusion in the school curriculum of information about the diversity of disorders and treatment modalities in psychiatry.

Leff, J. \& Trieman, N. (2000) Long-stay patients discharged from psychiatric hospitals. Social and clinical outcomes after five years in the community. The TAPS Project 46. British Journal of Psychiatry, I76, 217-223.

Creed, F. H., Black, D. \& Anthony, P. (1990)

Randomised controlled trial comparing day and inpatient psychiatric treatment. BMJ, 300, 1033-1037.

J. Leff Institute of Psychiatry, De Crespigny Park, London SE5 8AF, UK

\section{Use of outcomes measures by psychiatrists}

Gilbody et al (2002) highlight the poor adherence of psychiatrists to using instruments to measure clinical outcomes. Assessment tools and outcome measures have been in use among practitioners working with people with learning disability for many years. There are many validated tools and reliable measures available for use in clinical practice that are routinely used. The take-up of assessment tools and outcome measurements has perhaps been influenced by the proportion of this patient group who have poor verbal skills, making access to their mental state and internal world a challenge to the clinician.

Observation of behaviour is an important element of assessment of mental health problems in people with learning disabilities. The Mini Psychiatric Assessment Schedule for Adults with Developmental Disability (PAS-ADD) is commonly used to detect psychopathology in people presenting with challenging behaviour that may be due to mental illness. It has been shown to have good reliability and validity (Prosser et al, 1998). The Health of the Nation Outcome Scales for People with Learning Disabilities (HoNOS-LD) is a useful tool to measure change over time to a therapeutic intervention (Roy et al, 2002). Clinical observations can be carried out by any clinician in the multi-disciplinary team trained in the application of these clinical tools.
It could be argued that the use of these instruments is reductionist and does not communicate the breadth of human experience and suffering of patients. Where language fails to express the impact of mental illness and social distress, I would hold that the use of rating scales can objectively indicate the nature of the suffering and the effectiveness of interventions made in patient care.

I believe the key to the use of tools in the future will depend on educating trainees to use these instruments and allowing them to be freely available in clinical practice. Of course they would gain greater prominence in practice were they to form part of assessment in the MRCPsych examinations!

Gilbody, S. M., House, A. O. \& Sheldon, T. A. (2002)

Psychiatrists in the UK do not use outcomes measures. National Survey. British Journal of Psychiatry, $\mathbf{1 8 0}$, 101-103.

Prosser, H., Moss, S., Costello, H., et al (1998) Reliability and validity of the Mini PAS-ADD for assessing psychiatric disorders in adults with intellectual disability. Journal of Intellectual Disability Research, $\mathbf{4 2}$, 264-272.

Roy, A., Matthews, H., Clifford, P., et al (2002) Health of the Nation Outcome Scales for People with Learning Disabilities (HoNOS-LD). British Journal of Psychiatry, 180, 6I-66.

K. P. Courtenay Department of Psychiatry of Disability, St George's Hospital Medical School, London SWI7 ORE, UK

\section{Dissent as a symptom: why China has questions to answer}

In his letter taking issue with our claim that psychiatry is abused by the Chinese authorities for political control purposes, Dr Sing Lee (2001) cites his own experience in examining patients there suffering from 'qigong-related mental disorder'. $\mathrm{He}$ concludes that this culture-bound syndrome both exists and can be a serious condition, and that the psychiatric detention of Falun Gong practitioners in China today is therefore not a sign of the political abuse of psychiatry. Without challenging the validity of Chinese psychiatrists' diagnoses of qigong-related mental disorder in particular cases, it should be stressed that the Chinese authorities themselves hardly ever mention this diagnosis when justifying the psychiatric detention of Falun Gong practitioners. Indeed, recent articles in the Chinese psychiatric literature have stated 